Chiara Saraceno

\title{
Worte statt Taten
}

\section{Familienpolitik in Berlusconis Italien}

\section{Familienpolitik - eine Randnotiz der italienischen Geschichte}

Dass die Regierung Berlusconi keine Familienpolitik betreibt, ist in der neueren italienischen Geschichte keine Besonderheit. Im Gegenteil: Die Mitte-Rechts-Koalition steht in einer geradezu paradoxen Tradition, denn seit 1945 hat keine Regierung, ganz gleich welcher politischer Couleur, eigene Konzepte zur Förderung von Familien entwickelt ${ }^{1}$. Familienpolitik war in der zweiten Hälfte des 20. Jahrhunderts stets ein randständiges Phänomen. Lediglich in der ersten Amtszeit von Romano Prodi (1996 bis 1998) schafften es die Familien auf die politische Agenda. Wichtige Gesetzesvorhaben wie das Gesetz 285/2000, das den Aufbau sozialer Dienste für Kinder und Jugendliche vorsah, und das Gesetz 328/2000 über die Reform des Elternurlaubs fielen in diese Zeit. Die Elternzeit wurde seitdem flexibler gehandhabt; erstmals konnten auch erwerbstätige Väter einen Anspruch geltend machen. Letztlich blieb diese Flexibilisierung aber ein familienpolitisches Intermezzo. Nicht einmal alle Regierungsmitglieder unterstützten die Neuerungen. So befasste sich die Onofrio-Kommission, die Vorschläge zur Reform des italienischen Sozialstaats entwickeln sollte, nicht mit familienpolitischen Fragen. Die zweite Regierung Prodi (2006 bis 2008), die intern gespalten und politisch kaum handlungsfähig war, kümmerte sich fast gar nicht mehr um die Belange der Familien. Über diese Tatsache vermag auch die Schaffung eines eigenen Familienministeriums nicht hinwegzutäuschen; der zuständige Minister blieb nämlich ohne Geschäftsbereich².

\footnotetext{
${ }^{1}$ Vgl. Chiara Saraceno, Mutamenti della famiglia e politiche sociali in Italia, Bologna 2003.

${ }^{2}$ Vgl. Chiara Saraceno, Le politiche della famiglia, in: Luciano Guerzoni (Hrsg.), La riforma del welfare. Dieci anni dopo la „Commissione Onofri“, Bologna 2008, S. 399-418.
} 
Das bedeutet aber nicht, dass für Familien nichts getan würde und dass lediglich Stagnation zu beklagen sei ${ }^{3}$. Mutterschaftsurlaub etwa hat in Italien eine lange Tradition und ist, was Dauer und Höhe der Ansprüche anbelangt, sogar großzügiger geregelt als in den meisten anderen europäischen Staaten. So beziehen Mütter in Italien über fünf Monate ein Mutterschaftsgeld von mindestens 80 Prozent ihres früheren Gehalts. Anfangs beschränkt auf erwerbstätige Frauen in regulären Vollzeitjobs, wurde die Mutterschaftshilfe später auch auf Selbstständige und Frauen in zeitlich befristeten Arbeitsverhältnissen ausgeweitet. Eine weitere wichtige Veränderung bestand in der Einführung des Elternurlaubs in den 1970er Jahren, den auch Väter seit 1979 in beschränktem, ab 2000 dann in vollem Umfang in Anspruch nehmen durften. Lange Zeit waren die Konditionen in gewisser Hinsicht sogar günstiger als in Deutschland: Eltern hatten einen Anspruch auf eine zehnmonatige Freistellung und erhielten immerhin 30 Prozent des früheren Gehalts. Dies galt für alle abhängig Beschäftigten in einem regulären Arbeitsverhältnis ohne Ansehen des Einkommens. Da in Italien aber viele junge Menschen und Frauen jeden Alters in befristeten oder prekären Arbeitsverhältnissen beschäftigt sind, wären hier Reformen nötig, um die staatlichen Leistungen auch ihnen zukommen zu lassen; schließlich sind sie in dem Alter, in dem gemeinhin Kinder geboren werden.

Zwar ist der Anspruch auf Mutterschafts- beziehungsweise Elternurlaub nicht nur in Italien an die Erwerbstätigkeit der Eltern gebunden. Allerdings sind in kaum einem anderen Land die Zahlungen derart eng an abhängige Beschäftigungsverhältnisse und geringfügige Einkommen gekoppelt: Das Familieneinkommen muss zu 70 Prozent aus nichtselbstständiger Beschäftigung stammen und darf eine gewisse, an der Größe der Familie orientierte Höhe nicht überschreiten. Die italienische Familienpolitik ist somit Unterstützungspolitik für Geringverdiener, sofern diese in einem abhängigen Beschäftigungsverhältnis stehen. Sie trägt weder den faktischen Kosten für Kinder Rechnung, noch ist sie gegen echte Armut gerichtet, denn nur Beschäftigte haben Anspruch auf staatliche Unterstützung, Arbeitslose hingegen nicht. Von Armut betroffene Familien ohne eigenes Einkommen bleiben somit weitgehend

3 Vgl. Manuela Naldini/Chiara Saraceno, Social and family policies in Italy: not totally frozen but far from structural reforms, in: Social Policy \& Administration 42 (2008), S. 733-748. 
auf sich allein gestellt, sieht man von den Leistungen auf regionaler und kommunaler Ebene einmal ab.

Sozialstaatliche Leistungen wie soziale Dienste für Kleinkinder und gebrechliche alte Menschen fallen nahezu ausschließlich in die Kompetenz der Gemeinden. Im Unterschied etwa zum Gesundheitswesen macht der Staat weder gesetzliche Vorgaben über einzuhaltende Standards noch beteiligt er sich an den finanziellen Zuwendungen. Die Verfassungsreform von 2001 hat die Autonomie der politischen Peripherie - und hier vor allem der Regionen - sogar noch gestärkt, so dass diese etwa auch den Umfang der angebotenen Leistungen selbst bestimmen können. Im Prinzip müsste die Staat-RegionenKonferenz Mindeststandards für Hilfsleistungen definieren, auf deren Basis dann die finanzielle Verantwortung des Staates festzulegen wäre. Solche Festlegungen gibt es jedoch nicht. Gravierende Unterschiede zwischen den Regionen und Gemeinden in puncto Qualität und Versorgungsanspruch von hilfsbedürftigen alten Menschen und Kleinkindern sind die unausweichliche Folge; offensichtlich gilt hier das Prinzip des cuius regio eius religio. Die Frage der Zuwendungen für Kleinkinder wird mittlerweile öffentlich diskutiert und von Zeit zu Zeit auch in der Politik thematisiert. Dazu trägt sicher bei, dass inzwischen auch in Italien die Mehrzahl der Frauen mit Kindern im Vorschulalter vollzeitbeschäftigt ist. Ganz anders sieht es dagegen bei pflegebedürftigen alten Menschen aus. Ihnen stehen zwar finanzielle Hilfen zu, aber nur dann, wenn sie vollständig pflegebedürftig sind. Sie haben aber keinen Anspruch auf persönliche Pflege und Betreuung, selbst wenn diese Dienstleistungen vor Ort angeboten werden ${ }^{4}$. Als Konsequenz daraus hat sich in Italien ein neuer Markt für häusliche Altenpflege etabliert. Migrantinnen, die zumeist ohne regulären Arbeitsvertrag und damit auch ohne Ansprüche auf Sozialleistungen beschäftigt werden, bedienen die starke Nachfrage nahezu vollständig.

Grafik $1^{5}$ dokumentiert öffentliche Ausgaben für Kinder in Italien im Vergleich mit den diesbezüglichen Leistungen anderer europäischer Staaten; Italien schneidet dabei eher schlecht ab.

${ }^{4}$ Vgl. Francesca Bettio/Annamaria Simonazzi/Paola Villa, Change in care regimes and female migration: The „care drain“ in the Mediterranean, in: Journal of European Social Policy 16 (2006), S. 271-285.

5 Aufgrund der unterschiedlichen Verhältnisse in den EU-Staaten basiert der Vergleich auf Durchschnittszahlen. Die Höhe der Aufwendungen 


\section{Chiara Saraceno}

Grafik 1: Finanzielle Zuwendungen für Familien mit Kindern

Anteil des durchschnittlichen Nettoeinkommens

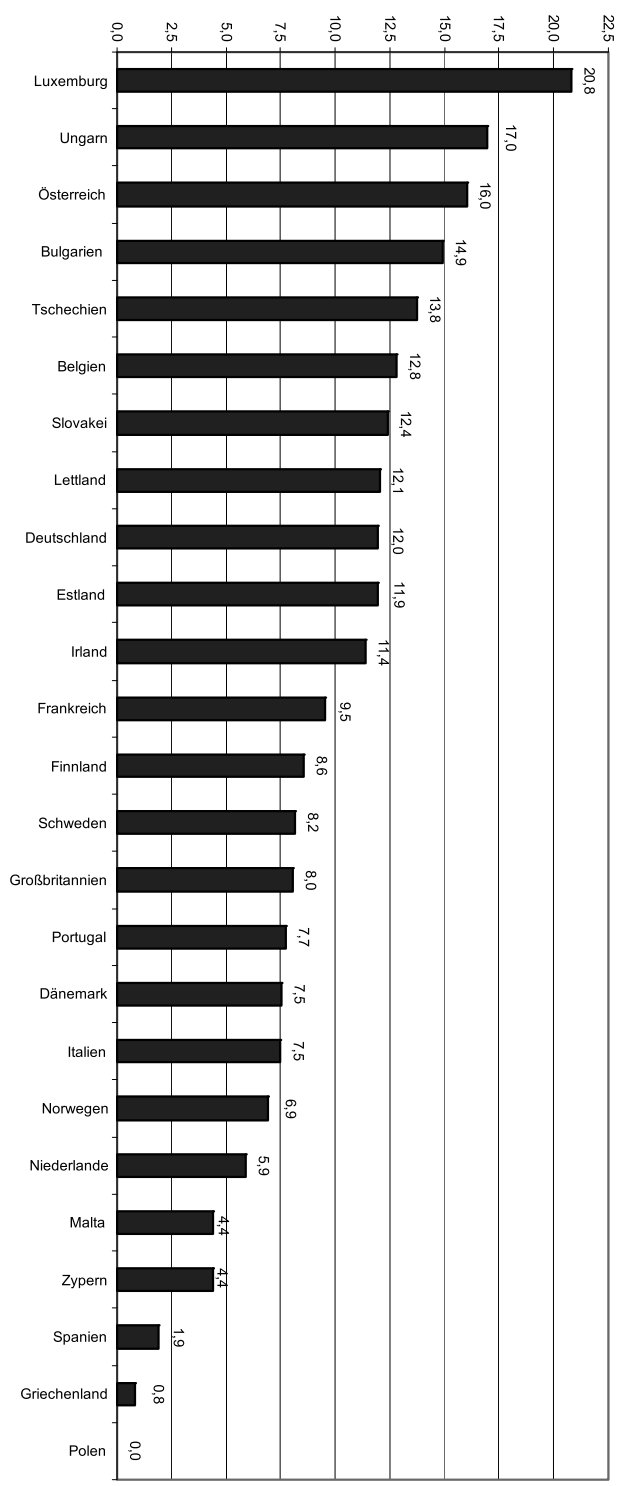

Quelle: Projekt Multilinks nach Daten von Eurostat. 
Hinzu kommt, dass der Staat Familien mit Kindern im Vorschulalter weitgehend im Stich lässt. Es bestehen zwar starke regionale Unterschiede, klar ist aber, dass häusliche Erziehung (hauptsächlich von Kindern unter drei Jahren) in Italien besonders groß geschrieben wird. Die Betreuung des Nachwuchses liegt häufig bei den Großeltern; ohne diese generationsübergreifende Unterstützung könnten viele junge italienische Mütter Kind und Beruf nicht miteinander vereinbaren.

Nicht viel besser steht es bei Hilfen der öffentlichen Hand für pflegebedürftige ältere Menschen, wie Grafik 2 zeigt. Die Daten zur Pflege alter Menschen sind lückenhaft und disparat, was auch daran liegt, dass die genaue Zahl der teilweise oder völlig pflegebedürftigen Personen (der Status wird in den EULändern unterschiedlich definiert) nicht immer bekannt ist. Grafik 2 vermittelt somit nur ein ungefähres Bild von der Zahl älterer Menschen über 65 Jahre, die zu Hause oder in Pflegeheimen und anderen sozialen Einrichtungen betreut werden. $\mathrm{Zu}$ beachten ist, dass in den letzten Jahren in allen EU-Staaten nicht nur die Betreuungsdienste für ältere Menschen stark ausgebaut, sondern zunehmend auch verschiedene Formen von Geld-für-Pflege eingeführt wurden. Auch auf diesem Feld rangiert Italien im internationalen Vergleich auf einem der unteren Plätze. Die Pflege liegt zu einem großen Teil bei den Familien oder muss auf dem freien Markt organisiert werden.

Ergänzt man diese mißlichen Umstände noch um die Tatsache, dass der soziale Wohnungsbau und die Sozialversicherung vor allem junge Menschen benachteiligt und sie in jahrelange Abhängigkeit von ihren Eltern bringt, wird eines überdeutlich: Der italienische Sozialstaat basiert zu einem ganz erheblichen Maß auf dem Prinzip der Familiensolidarität ${ }^{6}$. Das hat Folgen nicht nur für die Lebenschancen von Männern und Frauen innerhalb und außerhalb der Familien. Die dadurch beförderte soziale Ungleichheit wirkt sich auch auf die Gesellschaft als

wurde errechnet aus den direkten und indirekten Zuwendungen, die a) eine Familie mit zwei Kindern mit einem Einkommen erhält, das 133 Prozent über dem Durchschnittseinkommen liegt, sowie die b) eine Familie bekommt, die keine Kinder hat. Im italienischen Fall stammen die Einkommen überwiegend aus abhängiger Beschäftigung. Es fehlen Daten für Rumänien, Slowenien und Litauen.

${ }^{6}$ Vgl. Massimo Livi Bacci, Avanti, giovani. Alla riscossa, Bologna 2008; Naldini/Saraceno, Social and family policies in Italy; Daniela Del Boca/ Alessandro Rosina, Famiglie sole. Sopravvivere con un welfare inefficiente, Bologna 2009. 


\section{Chiara Saraceno}

Grafik 2: Anteil der älteren Menschen, die Zuschüsse der öffentlichen Hand zur stationären oder ambulanten Pflege erhalten, in Relation zur Bevölkerung, die älter als 65 Jahre ist

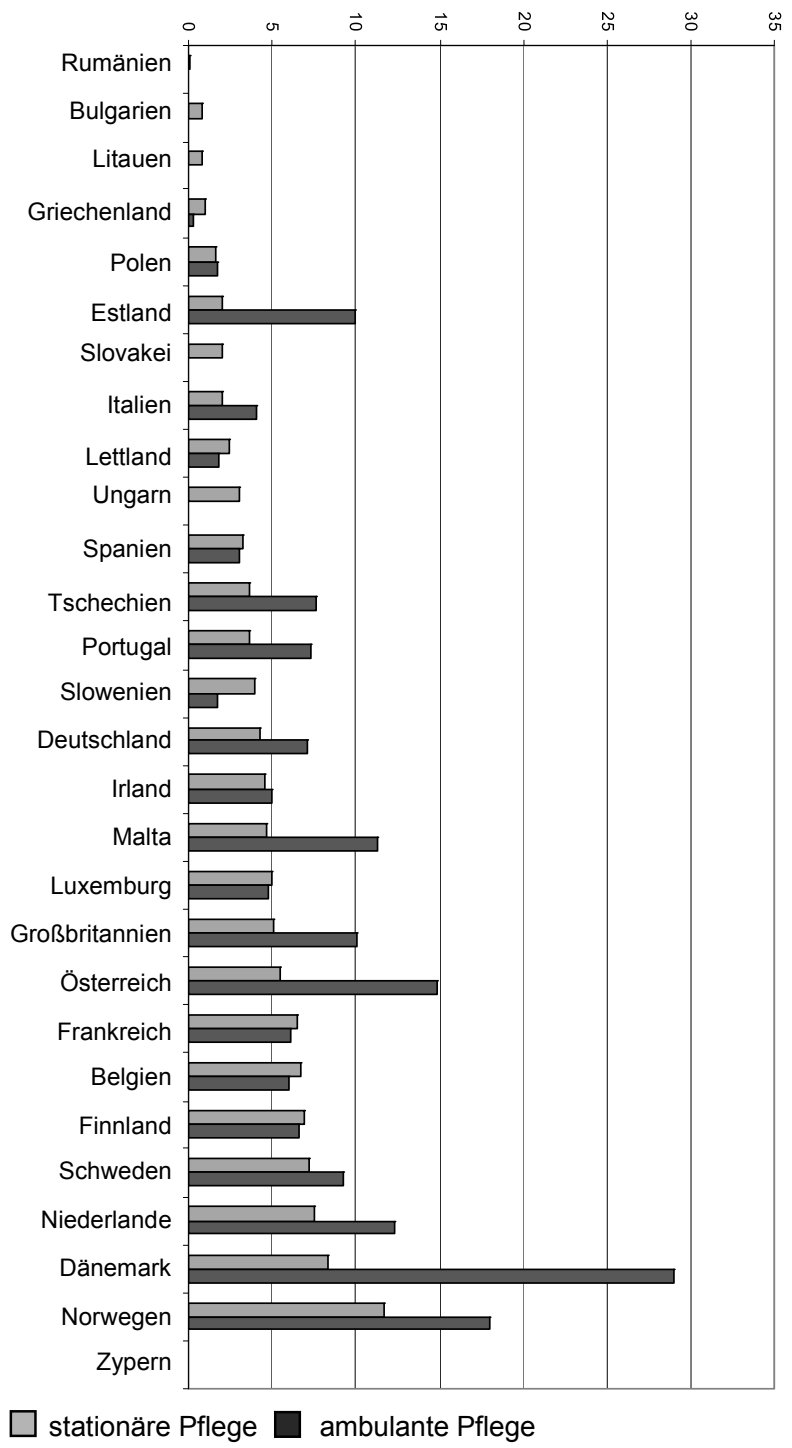

Quelle: Projekt Multilinks auf der Basis einer Vielzahl von Quellen. 
Ganzes aus, denn die soziale oder geschlechtliche Diskriminierung reproduziert sich von Generation zu Generation.

Es gibt viele Gründe für die permanente Marginalisierung der Familienpolitik in Italien: Bis in die 1980er Jahre scheute man sich, hier neue Akzente zu setzen, um nur ja nicht in die Nähe faschistischer Praktiken gerückt zu werden. Als besonders anrüchig galten die pronatalistischen Maßnahmen des Regimes, die eine Hebung der Geburtenrate bewirken und Italien so in die Lage versetzen sollten, ein Imperium zu erobern, sowie die penetrante politische Instrumentalisierung der Familien, die ganz in den Dienst der Perpetuierung faschistischer Werte und Weltanschauungen gestellt wurden. Die Konsequenz war, dass Artikel 29 der neuen demokratischen Verfassung von 1948 keinen Zweifel daran ließ, dass der Staat die Familie als „natürliche, auf die Ehe gegründete Lebensgemeinschaft“ zu schützen habe. Obwohl diese Bestimmung heute gerne von Gegnern gleichgeschlechtlicher Partnerschaften ins Feld geführt wird, um ihre Position zu untermauern, kann niemand darüber hinwegsehen, dass die Verfassungsväter vor allem den Schutz der Familie vor staatlichen und politischen Eingriffen im Auge hatten; hier liegt übrigens eine bemerkenswerte Parallele zum Grundgesetz der Bundesrepublik Deutschland ${ }^{7}$. Dass Artikel 29 im Laufe der Geschichte eher dazu benutzt wurde, alternative Formen von Familie zu delegitimieren als konkrete Hilfsprogramme für traditionelle Familien zu begründen, hat aber auch damit zu tun, dass man weiblicher Erwerbstätigkeit lange Zeit skeptisch gegenüberstand. Italien war ein Land mit niedriger männlicher Erwerbsquote, das seit jeher auf finanzielle Transferleistungen setzte und die Infrastruktur für staatliche Dienstleistungen vernachlässigte. Weibliche Berufstätigkeit galt in dieser Perspektive als Verlustgeschäft, weil sie die unbezahlten familiären und nachbarschaftlichen Hilfen und damit das ganze Unterstützungssystem gefährdete.

Auch die bewährte, in vielerlei Hinsicht unumgängliche Familiensolidarität hat dazu beigetragen, die Nachfrage nach sozialen Dienstleistungen gering zu halten. Hier scheint sich allerdings angesichts der fortschreitenden Überalterung der Gesellschaft eine Trendwende anzubahnen. Die Solidarität in

7 Vgl. dazu Ilona Ostner/Chiara Saraceno, Keine Arbeit, keine Kinder, keine Lösung? Italien und Deutschland in vergleichender Perspektive, in: Bruno Cattero (Hrsg.), Modell Deutschland - Modell Europa. Probleme, Perspektiven, Opladen 1998, S. 183-205. 
den Familien ist - nicht zuletzt aufgrund der demographischen Wende - auch in Italien neuen Belastungen ausgesetzt, wie die wachsende Bedeutung von Immigrantinnen auf dem Feld der Altenpflege zeigt. Schließlich darf nicht vergessen werden, dass die Familie seit der Staatsgründung ein heftig umkämpftes Streitobjekt zwischen Kirche und Staat war. Was ist eine Familie, welche familiären und sexuellen Gepflogenheiten sind sozial akzeptabel? Wer ist berechtigt, darüber zu richten? Diese Fragen sind seit Anfang der 1990er Jahre brennender geworden. Das lag an der Pluralisierung der familiären Lebensstile, aber auch an den Fortschritten in der Reproduktionsmedizin, die bisher unbekannte ethische Fragen aufgeworfen haben.

Die Familie ist mithin Gegenstand einer öffentlichen Debatte geworden, die ebenso großes Mobilisierungspotential besitzt wie ähnliche Auseinandersetzungen in den 1970er Jahren, als zunächst das Recht auf Scheidung und dann das Recht auf Abtreibung durchgesetzt wurden. Die Sensibilität des Themas hat Lösungen freilich eher erschwert. Staat und politische Parteien haben sich hier ja nicht nur mit der katholischen Kirche als steinernem Gast auseinanderzusetzen. Im Sozialstaatsmodell, das sich in Italien in der zweiten Hälfte des 20. Jahrhunderts herausgebildet hat, gibt es für sie in Gestalt der Gewerkschaften einen weiteren einflussreichen Gegenspieler. Die Gewerkschaften vertreten vor allem die Interessen der abhängig Beschäftigten, die ihrerseits im Zentrum des Arbeitsmarkts stehen. Obwohl der Feminismus mittlerweile auch die Gewerkschaften erreicht hat, spielen Fragen wie Gleichberechtigung und Gleichstellung nur eine untergeordnete Rolle. Auch die verlängerte Abhängigkeit junger Erwachsener von ihren Eltern wird kaum thematisiert. Weil die Gewerkschaften darüber hinaus ihre Kernklientel auch dann unterstützen, wenn sie bereits Rente beziehen, sind sie verständlicherweise an einer Umverteilung der Soziallasten zum Nachteil der Rentner und zugunsten von Familien wenig interessiert.

\section{Berlusconi und der "Schutz der Familie"}

Die staatliche Untätigkeit in der Familienpolitik, die auch die Ära Berlusconi kennzeichnet, ist umso bedenklicher, als in den letzten Jahren tiefgreifende Veränderungen in der Gesellschaft zu beobachten sind. Zu nennen sind hier vor allem die wachsende Zahl berufstätiger Frauen und die zunehmende Überalterung der Bevölkerung. Berlusconi antwortete darauf mit einem 
dreifachen Widerspruch zwischen Anspruch und Wirklichkeit, der zum Signum seiner Familienpolitik geworden ist ${ }^{8}$.

Da ist erstens die Tatsache, dass die Regierung Berlusconi zwar immer den „Schutz der Familie“ predigte, über bloße Worte aber nie hinauskam. Das Hohelied auf den Wert der traditionellen Familie diente zumeist politischen Propagandazwecken, etwa wenn es darum ging, sich der Unterstützung der katholischen Kirche zu versichern. Besonders deutlich wurde das in der Diskussion um Homosexualität oder in der Debatte um die Bedeutung der Ehe als der angeblich einzig legitimen Basis der Familie. Dabei verbinden sich zuweilen Bevölkerungs- und Ausländerpolitik, da sogar Einwanderern mit Aufenthaltsgenehmigung staatliche Leistungen vorbehalten bleiben, die eingeführt wurden, um die Zahl der Geburten zu erhöhen.

Daran schließt sich der zweite Widerspruch an: Trotz der Betonung des Werts der Familie für die Gesellschaft kann die Regierung nicht einmal mit brauchbaren Förderinstrumenten für traditionelle Familien aufwarten: Allgemeine Zulagen für Familien mit Kindern existieren nicht, die finanzielle Unterstützung für den bezahlten Elternschaftsurlaub blieb eingefroren, Steuerreformen brachten keine Entlastung. Stattdessen hat die Regierung Berlusconi mit zahlreichen Gesetzen auf geradezu obsessive Weise in die Intimsphäre und die persönlichen Freiheiten der Italiener eingegriffen: bei der Frage der künstlichen Befruchtung ebenso wie bei der Abtreibung und der Sterbehilfe.

Schließlich gibt es drittens einen tiefgreifenden Widerspruch zwischen den Versprechungen vor der Wahl und der Politik danach. Berlusconi hatte im vergangenen Wahlkampf zwar feierlich verkündet, dass Italien binnen kurzem mit einer Kehrtwende in der Steuerpolitik rechnen könne und dass nach dem Vorbild des französischen Familienquotienten nicht mehr der Einzelne, sondern die Familie zur Bemessungsgrundlage würde. Bisher sind diese Versprechungen allerdings nur gebetsmühlenartig wiederholt, aber nicht einmal ansatzweise realisiert worden. Dabei fürchtet die Regierung weniger negative Auswirkungen auf die Frauenerwerbstätigkeit als hohe Kosten in der Form

8 Ein Überblick über die Entwicklung der Sozialpolitik und die soziale Lage in Italien findet sich bei Maurizio Ferrera, Le politiche sociali. L'Italia in prospettiva comparata, Bologna 2006. In deutscher Sprache zu empfehlen: David Natali, Rekalibrierung von Sozialprogrammen und Flexibilisierung der Arbeitsmarktpolitik: Das italienische Wohlfahrtssystem, in: Klaus Schubert/Simon Hegelich/Ursula Bazant (Hrsg.), Europäische Wohlfahrtssysteme. Ein Handbuch, Wiesbaden 2008, S. 333-353. 
ausbleibender Steuereinnahmen. Paradoxerweise blieb so ein Steuersystem unangetastet, das seine eigentlichen Träger, die Familien, besonders stark belastet.

Diesen Widersprüchen stehen zwei Initiativen gegenüber, die man zumindest vordergründig unter Familienförderung rubrizieren kann: die Abschaffung der Gemeindesteuer auf den Hauptwohnsitz in Privateigentum und die Legalisierung des Aufenthalts von ausländischen Haushaltshilfen, Kindermädchen und Pflegekräften. Bei der Steuerbefreiung setzte Berlusconi den Kurs der Vorgängerregierung Prodi fort, die allerdings nur Familien mit geringem Einkommen begünstigt hatte. Berlusconi ging es aber gerade nicht um Umverteilung, denn der Steuerfreibetrag wurde nicht an den Wert der Immobilie gekoppelt und die Größe der Wohnung nicht mit der Zahl der Bewohner in Beziehung gesetzt. Letztlich wurden damit alle über einen Kamm geschoren, wobei die Besitzer großer Immobilien am meisten profitierten.

Auf der anderen Seite mussten die Gemeinden durch den Wegfall der Steuer beträchtliche finanzielle Einbußen verkraften und waren deshalb nicht mehr in der Lage, die üblichen Familienhilfen - vor allem die Unterstützung von Kindern und älteren Menschen - in der alten Höhe aufrechtzuerhalten. Zwar hatte der Staat versichert, die Einbußen der Kommunen durch Ausgleichszahlungen zu kompensieren. Diese Ankündigung blieb jedoch wegen strapazierter Kassen ein leeres Versprechen und provozierte deshalb heftige Beschwerden und Demonstrationen des italienischen Gemeindetags, die allerdings zu keinem Sinneswandel führten. Den Familien wurde also auf der einen Seite etwas gegeben, auf der anderen Seite aber gleich viel genommen. Das Nachsehen aber hatten wieder einmal Familien mit geringeren Einkommen beziehungsweise Familien mit kleinen Kindern oder unterhaltspflichtigen Jugendlichen; bei ihnen fällt die Bilanz letztlich sogar negativ aus.

Die Maßnahmen zugunsten ausländischer Haushaltshilfen, Kindermädchen und Pflegekräfte wurden 2009 im Rahmen einer Verschärfung des Einwanderungsgesetzes ergriffen. Hier handelt es sich um Familienpolitik sui generis, die allerdings erst auf massiven Druck der Öffentlichkeit initiiert wurde: Viele Italiener sahen in der Beschäftigung ausländischer Hilfskräfte die einzige Möglichkeit, ihre pflegebedürftigen Angehörigen zu Hause zu versorgen, und erhoben Protest, als eine neue Rechtslage diese Möglichkeit zu verbauen drohte. Mit Erfolg - die Familien, die unter der Hand ausländische Pflegekräfte beschäf- 
tigten, konnten diese nicht selten ohne Aufenthaltsgenehmigung im Land lebenden Personen nun regulär anstellen. Die Kosten für die Legalisierung müssen freilich die Familien tragen, und sie sind es auch, die Intensität und Qualität der Dienstleistungen zu kontrollieren haben. Aufgrund der hohen Kosten und einiger Besonderheiten der Legalisierung, die letztlich nur dauerhaft bei einer Familie tätigen Ausländerinnen offensteht, hat sich bisher anscheinend nicht einmal die Hälfte der illegal arbeitenden ausländischen Haushaltshilfen bei den Behörden gemeldet $^{9}$. Die Frage der illegalen Beschäftigungsverhältnisse ist damit ebenso wenig gelöst wie die der Familienlasten.

Schließlich verstößt eine Initiative der Regierung Berlusconi eindeutig sowohl gegen das Vorhaben, Familie und Beruf unter einen Hut zu bringen, dem sie sich unter Beachtung europäischer Kriterien selbst verschrieben hat, als auch gegen den Vorsatz, gleiche Lebenschancen für Kinder und Jugendliche zu schaffen, unabhängig von ihrer sozialen Herkunft und ihrem Wohnort. So ist die Unterrichtszeit an italienischen Grundschulen verkürzt und der bisherige Ganztagsunterricht in eine Art Nachmittagsbetreuung umgewandelt worden, deren Kosten die Eltern zu tragen haben. Betroffen hiervon sind insbesondere Familien, in denen Mutter und Vater oder der allein erziehende Elternteil arbeiten; ihr Leben ist damit noch stressiger und teurer geworden. Am schlimmsten ist die Situation in Süditalien, wo der Ganztagsunterricht das einzige Mittel zu sein scheint, die aus der grassierenden Armut resultierenden, auch in den PISA-Studien belegten schulischen Defizite der Kinder auszugleichen.

\section{Schlussbemerkungen}

Wie seine Vorgänger betreibt auch Berlusconi keine genuine Familienpolitik. In Zeiten allerdings, in denen sich die italienische Gesellschaft der Erosion der traditionellen Familie und einer zunehmenden Überalterung ausgesetzt sieht, zieht diese Untätigkeit weitreichende Konsequenzen nach sich, zumal auch die Frauenerwerbstätigkeit zunimmt. Da der Staat die Augen verschließt, dürften schon in absehbarer Zeit nicht mehr alle Kinder und pflegebedürftigen Alten vernünftig versorgt werden können.

${ }^{9}$ Hinzu kommt, dass die Haushaltshilfen allein auf Vollzeitbasis bei einer einzigen Familie beschäftigt werden müssen. Privilegiert werden dadurch vor allem wohlhabende Familien. 
Die seit Jahren konstant niedrige Geburtenrate ist ein eindeutiger Indikator für diese Probleme. Trotzdem scheint die Regierung paralysiert zu sein. Im Weißbuch zur Zukunft des Sozialstaats fällt ihr nach einer eingehenden Analyse nichts anderes ein, als lapidar an die Solidarität der Familien zu appellieren und zu mehr freiwilliger Arbeit aufzurufen. Das steht nicht nur in krassem Widerspruch zu den Bedürfnissen der Familien und hilft wenig gegen die Gefahr generationell reproduzierter Ungleichheit. Diese Ignoranz widerspricht auch dem Aktivismus, den die Regierung Berlusconi beweist, wenn es nicht um Fragen der Umverteilung, sondern um gesetzliche Neuregelungen geht, die Familiengründungen und innerfamiliäre Beziehungen konditionieren und überhaupt die bürgerlichen Freiheiten beschränken. Dieser moralisch motivierte Aktivismus schert sich wenig um die Gepflogenheiten einer pluralistischen Demokratie und zielt vor allem darauf, den Beifall und die Unterstützung der katholischen Kirche zu gewinnen.

Nicht umsonst besuchte Berlusconi nach seiner Amtseinführung 2008 sogleich den Vatikan, wo er offiziell erklärte, dass seine Regierung in Fragen der Familienpolitik und der Bioethik den Vorstellungen der Kirche entsprechen werde. Diese Übereinkunft wurde weiter bekräftigt und ist nach den zahlreichen Sexskandalen des Ministerpräsidenten 2009 sogar zu einer Art Tauschgeschäft geworden, das sich auf einen einfachen Nenner bringen lässt: die Kirche hält sich mit Kritik zurück, was Berlusconi auf seine Weise vergilt.

Ganz ähnliche „Pakte“ haben seine Koalitionspartner geschlossen: Die Lega Nord beispielsweise hat sich den Schutz der traditionellen Familie und der christlichen Symbole im öffentlichen Raum auf ihre Fahnen geschrieben, um auf diese Weise gegenüber der katholischen Kirche Boden gut zu machen, die sich von der rassistischen und ausländerfeindlichen Rhetorik der Lega irritiert zeigte. Die Familie ist damit stärker als je zuvor zum Tauschobjekt der Mächtigen geworden, hat aber selbst am wenigsten davon. Die staatlichen Leistungen, die ihr zustehen, sind gering, während die Erwartungen, die man von den verschiedensten Seiten an sie heranträgt, immer größer werden. Die Politik - allen voran Berlusconi - beschränkt sich in Zeiten leerer Kassen auf hehre Worte, denen aber nur selten Taten folgen.

Aus dem Italienischen übersetzt von Patrick Bernhard. 